\title{
Correction to: Integrating Mathematics and Science Within STEM Paradigm
}

\section{Correction to:}

\author{
A. Sokolowski, Scientific Inquiry \\ in Mathematics - Theory and Practice \\ https://doi.org/10.1007/978-3-319-89524-6_2
}

The chapter was inadvertently published with an error in the figure 2.3 and 2.4. The captions were incorrect and they have been corrected as follows:

Fig. 2.3 should be labelled as "Process of deductive inquiry"

Fig. 2.4 should be labelled as "Process of inductive inquiry" 